\title{
Analysis of holographic photopolymers for integrated optical systems via quantitative phase microscopy
}

David J. Glugla, Marvin D. Alim, Madeline B. Chosy, Amy C. Sullivan, Robert R. McLeod

David J. Glugla, Marvin D. Alim, Madeline B. Chosy, Amy C. Sullivan, Robert R. McLeod, "Analysis of holographic photopolymers for integrated optical systems via quantitative phase microscopy," Proc. SPIE 10233, Holography: Advances and Modern Trends V, 102330I (15 May 2017); doi: $10.1117 / 12.2265873$

SPIE. Event: SPIE Optics + Optoelectronics, 2017, Prague, Czech Republic 


\title{
Analysis of holographic photopolymers for integrated optical systems via quantitative phase microscopy
}

\author{
David J. Glugla ${ }^{\mathrm{a}}$, Marvin D. Alim ${ }^{\mathrm{b}}$, Madeline B. Chosy ${ }^{\mathrm{c}}$, Amy C. Sullivan ${ }^{\mathrm{a}}$, Robert R. Mcleod ${ }^{\mathrm{a}}$
}

${ }^{a}$ Department of Electrical Engineering, University of Colorado, 425 UCB, Boulder, CO USA 80309; ${ }^{\mathrm{b}}$ Material Science and Engineering Program, University of Colorado, $596 \mathrm{UCB}$, Boulder, CO USA 80309; ' Department of Chemistry, Carleton College, 1 North College Street, Northfield, MN USA 55057

\begin{abstract}
Optically-driven diffusion of high refractive index molecules within a transparent thermoset polymer matrix is a promising platform for hybrid optics that combines a wide range of optical structures from large scale holograms to micron-scale gradient index waveguides in a single integrated optical system. Design of such a system requires characterization of the optical response of the material at a wide range of spatial scales and intensities. While holographic analysis of the photopolymers is appropriate to probe the smaller spatial scales and lower intensity optical response, quantitative phase mapping of isolated structures is needed to probe the response to the higher intensities and lower spatial frequencies used in direct write lithography of waveguides. We apply the transport of intensity equation (TIE) to demonstrate quantitative refractive index measurements of $10 \mu \mathrm{m}$-scale localized gradient index structures written into diffusive photopolymer materials using both single- and two-photon polymerization. These quantitative measurements allow us to study the effect of different exposure conditions and material parameters such as writing beam power, exposure time, and $\mathrm{wt} \%$ loading of the writing monomer on the overall profile of the refractive index structure. We use these measurements to probe the time scales over which diffusion is significant, and take advantage of the diffusion of monomer with a multiple-write scheme that achieves a peak refractive index contrast of 0.025 .

Keywords: transport of intensity, holography, photopolymer, waveguide, diffusion, integrated optics
\end{abstract}

\section{INTRODUCTION}

Two-component photopolymer gels offer a low-cost, versatile material system that incorporates the high diffusivity and reaction rates associated with liquid chemistry into a solid, robust polymer package. This form factor has proven effective in the creation of numerous embedded and diffractive optical devices, ranging from optical waveguides and flat optics to holographic displays, and solar concentrators $[1,2,3,4]$. The diverse library of available monomers and polymers allows for extensive customization of the material properties, while the orthogonal polymerization chemistries of the initial thermoset matrix and the optically-activated writing monomer allow for distinct control over the mechanical and optical properties of the final product.

While there are successful commercial options available, such as Covestro's Bayfol material [5], fundamental materials research can create systems that are customizable for specific applications, designing for such properties as high change in refractive index, specific wavelength response ranges and absorption spectra, various mechanical properties, ease or speed of processing, or biocompatibility. As new materials are developed, it is essential to be able to fully assess their optical properties over a wide range of spatial and intensity scales. Design of materials systems requires characterization of the optical response of the material at spatial scales ranging from 100s of nanometers for reflection holograms, to millimeters for larger optical elements. The optical response must also be probed over a wide range of intensities, from $\mathrm{mW} / \mathrm{cm}^{2}$ for large area exposures by low power, continuous wave lasers for holography up to $\mathrm{MW} / \mathrm{cm}^{2}$ (average power) for focused exposures from high power, pulsed lasers for two-photon direct write of isolated waveguides.

While holographic analysis of the photopolymers is appropriate to probe the smaller spatial scales and lower intensity optical response, it can only probe a single spatial frequency set by the hologram pitch and does not provide a full quantitative mapping of the index response. Quantitative phase imaging of isolated structures is needed to probe the response to the higher intensities of focused pulsed lasers used to write waveguides. Popular techniques such as ShackHartman wavefront sensing and interferometry either lack the resolution necessary to study isolated micron-scale structures or are sensitive to phase aberrations within the optical system. The transport of intensity equation (TIE)

Holography: Advances and Modern Trends V, edited by Miroslav Hrabovský, John T. Sheridan, Antonio Fimia, Proc. of SPIE Vol. 10233, 102330I · C 2017 SPIE · CCC code: 0277-786X/17/\$18 · doi: 10.1117/12.2265873 
derived by Teague in 1983, is a non-interferometric imaging technique that directly recovers the optical phase delay by using the axial change in intensity of a series of defocused images about the object plane [6]. Because the TIE does not suffer from strict coherence requirements of the illumination source, it may be implemented in a simple brightfield microscope $[7,8]$. Given the thickness of the phase structure, the TIE can be used to directly map the refractive index contrast of a 2D phase element and has been used to study transparent phase structures such as cells and waveguides fabricated in glass $[9,10,11]$.

We apply the TIE to demonstrate quantitative index measurements of localized gradient index structures written into diffusive photopolymer materials using both single- and two-photon polymerization. These quantitative measurements allow us to study the effect of different exposure conditions and material parameters such as writing beam power, exposure time, and $\mathrm{wt} \%$ loading of the writing monomer on the overall profile of the refractive index structure. We measure characteristic diffusion times of writing monomer along with refractive index, and take advantage of the diffusion of monomer with a multiple write system that achieves a peak refractive index contrast of 0.025 . These phase microscopy measurements, along with holographic analysis of these materials, will allow us to design optical systems including both large-scale holograms along with localized structures such as waveguides.

\section{EXPERIMENTAL METHODS}

\subsection{Materials}

The "liquid" components of the photopolymer gel commonly include a photoinitiator and monomer encapsulated within a solid, crosslinked polymer network via an orthogonal polymerization reaction. Photoexposure initiates polymerization, locally consuming the monomer and creating immobile polymer chains. Subsequent diffusion causes the monomer concentration to re-equilibrate, after which a final uniform light exposure polymerizes the remaining monomer and renders the material optically inert.

The writing monomer 1a (phosphorothioyltris(oxybenzene-4,1-diylcarbamoyloxyethane-2,1-diyl) triacrylate was synthesized following a procedure reported in various Covestro patent literature [12]. Briefly, $0.0207 \mathrm{~g}(0.09 \mathrm{mmol})$ of 2,6-di-tert-butyl-4-methylphenol and $57 \mathrm{~mL}$ of Desmodur RFE (27\% solution of tris(p-isocyanatophenyl) thiophosphate in ethyl acetate, $38.9 \mathrm{mmol}$ ) were added to a dried $100 \mathrm{ml}$ round bottom flask with a magnetic stir bar. The reaction was started by adding $13.5 \mathrm{~g}(116.3 \mathrm{mmol})$ of 2-hydroxyethyl acrylate to the flask dropwise. The reaction was performed at $60^{\circ} \mathrm{C}$ with a reflux condenser overnight. The product was collected by cooling and removal of ethyl acetate in vacuum to obtain a waxy semicrystalline solid.

The crosslinked matrix of the two-component photopolymer system consists of a stoichiometric ratio of isocyanate (Desmodur N3900) and alcohol (polycaprolactone-block-polytetrahydrofuran-block-polycaprolactone) that forms a flexible polyurethane. The photoreactive component contains of a 1:10 molar ratio of photoinitiator TPO $(2,4,6-$ Trimethylbenzoyl-diphenyl-phosphineoxide) and 1a monomer described above. Samples were made with $10 \mathrm{wt} \%$ or 30 $\mathrm{wt} \%$ of this writing monomer. The matrix and photoreactive components were mixed together in their liquid form prior to significant polymerization of the polyurethane, degassed, and then cast between a $1 \mathrm{~mm}$ glass microscope slide and a $150 \mu \mathrm{m}$ glass coverslip. Sample thickness was set using spacers ranging between 13 and $25 \mu \mathrm{m}$. Polymerization occurred overnight in an oven at $60^{\circ} \mathrm{C}$.

\subsection{Exposure system}

The optical layout for the exposure system is shown in Figure 1. The sample is mounted on a 5-axis stage that controls both tip/tilt and xyz motion, while a confocal reflection microscope operating at $660 \mathrm{~nm}$ is used to align the sample and provide a non-contact method for measuring the thickness of the sandwiched photopolymer layer. The confocal beam is co-aligned with both a $405 \mathrm{~nm}$ continuous wave laser and a $780 \mathrm{~nm}$ pulsed laser (100 fs pulses with $50 \mathrm{MHz}$ repetition rate) that are used to initiate polymerization with one and two photons respectively. Because the chosen photoinitiator is non-absorptive at $660 \mathrm{~nm}$, the confocal arm does not initiate significant polymerization. The co-alignment of the writing and analyzing wavelengths allows for optical path length measurements directly where the exposures will occur. The 
known refractive index of the photopolymer, measured with a prism coupler, is used with this optical path length measurement to calculate the thickness of the sample. This thickness measurement is necessary for reconstructing the refractive index change via phase imaging.

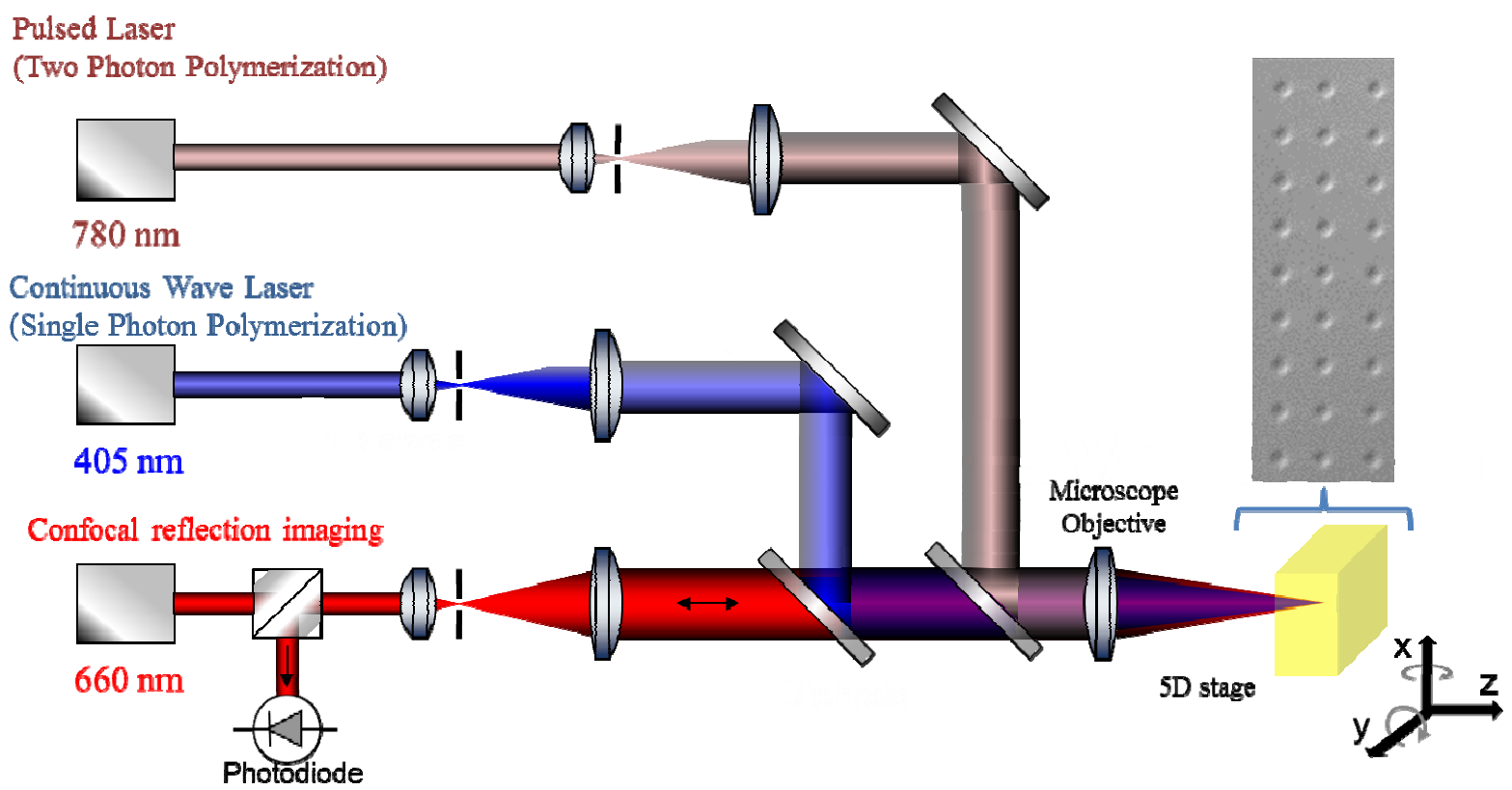

Figure 1. Optical scanning and exposure system. A $660 \mathrm{~nm}$ confocal reflection arm is used to locate and align the sample as well as measuring the thickness of the samples. The $405 \mathrm{~nm}$ and $780 \mathrm{~nm}$ exposure arms write index structures in the samples with one and two photons respectively. A Differential Interference Contrast image of rows of dots written into the material is shown to demonstrate the method.

Once the thickness of the sample has been determined, the $405 \mathrm{~nm}$ or $780 \mathrm{~nm}$ light is used to write a series of isolated points into the material using a range of intensities and exposure times. The write beam $1 / \mathrm{e}^{2}$ intensity diameter is $10 \mu \mathrm{m}$ when writing with $405 \mathrm{~nm}$, and $5.3 \mu \mathrm{m}$ when writing with $780 \mathrm{~nm}$. The beam power is adjusted to achieve a range of intensities, and multiple points are written for each exposure condition to provide error bars.

\subsection{Phase Imaging}

The refractive index of the samples is measured using a non-interferometric phase imaging technique based on the solution of the transport of intensity (TIE) equation developed by Teague [6] and shown in Eq. 1.

$$
\frac{\partial I(x, y)}{\partial z}=\frac{-\lambda}{2 \pi} \nabla_{x, y}\left(I(x, y) \nabla_{x, y} \phi(x, y)\right)
$$

where $I$ is the intensity distribution perpendicular to the optical axis along $z, \lambda$ is the wavelength of the incident light, and $\phi$ is the phase delay along the optical axis. The only information required to directly recover the two-dimensional (2D) optical path length through a phase structure is the axial change in intensity of light as it passes through the object. A second-order approximation of this derivative is calculated using a pair of symmetrically defocused images about the image plane. The detector noise in this approximation sets a limit on the minimum defocus distance, while the error associated with the second-order finite difference approximation imposes a competing limit on the maximum defocus distance $[13,14]$. Although there exists a variety of techniques to reduce this noise, most commonly by acquiring larger sets of defocused images [15,16,17], a single pair of defocused images is used in the experiments described here. All imaging is performed with a conventional brightfield microscope equipped with a 20x, 0.5 NA objective and a $633 \mathrm{~nm}$ line filter to prevent the light source from causing further reaction in the material. A defocus distance of $+/-3 \mu \mathrm{m}$ around the object plane using a piezo actuation is found to provide the best experimental results for the index structures studied in this paper. 
Fast Fourier Transforms (FFTs) are used to solve the TIE and so periodic boundary conditions are assumed. While the addition of an aperture at the image plane can be used to bypass this assumption, the imaged phase structures in our experiments are sufficiently isolated such that periodic boundaries can be ignored when solving the TIE [18]. Because the depth of field of the writing illumination is significantly larger than the thickness of the sample, and the absorption of the sample is insignificant over these thicknesses, the index of refraction of the written phase elements should be uniform throughout the thickness of the sample. Therefore, the thickness measurement from the confocal reflection microscope can be used to calculate the refractive index profile of the phase elements from the measured optical path length profile. An example of the quantitative refractive index structure calculated from a set of brightfield measurements in shown in Figure 2 .
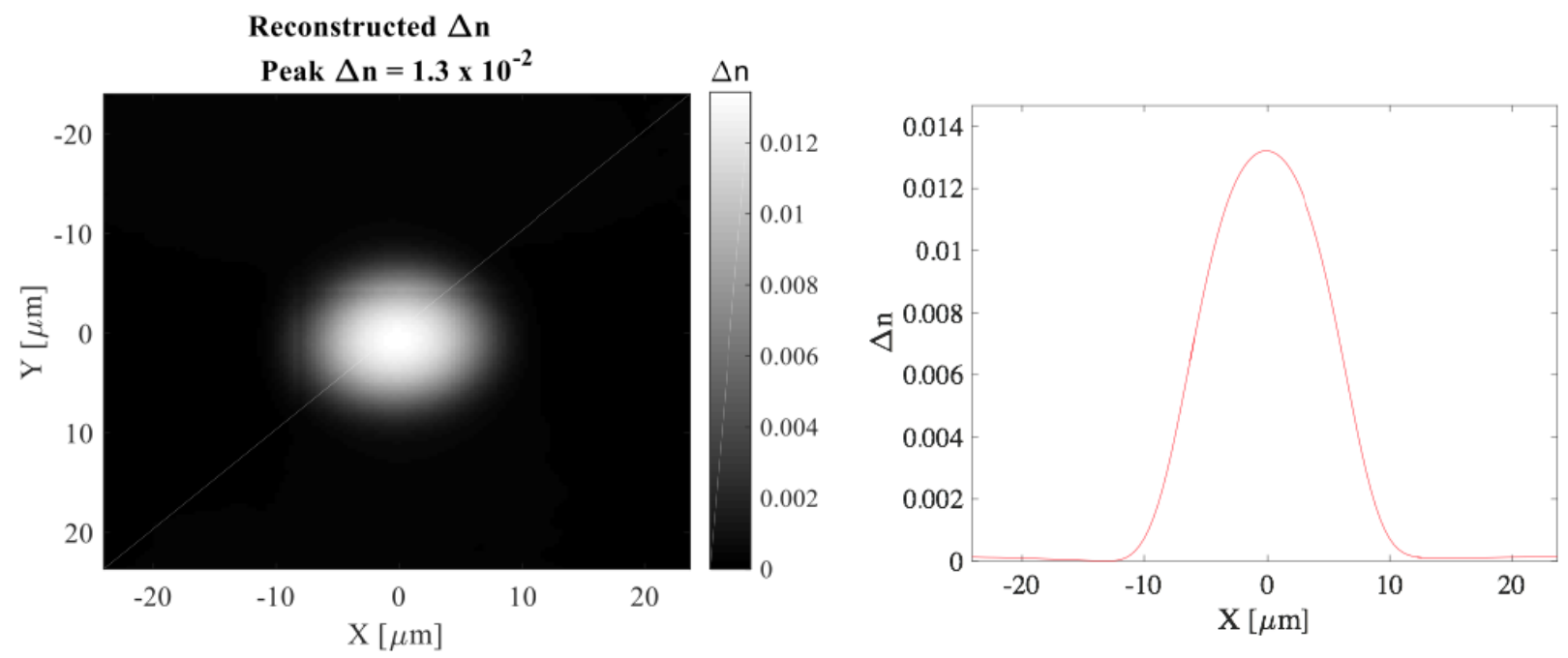

Figure 2. Example of the 2D refractive index profile, and a representative cross section calculated from brightfield microscope images and the transport of intensity equation. The peak value of $\Delta \mathrm{n}$, here 0.013 , is monitored vs. exposure conditions in the results described below.

\section{RESULTS}

\subsection{Single Photon Excitation}

The above writing and imaging systems are used to explore the response of the photopolymer under single-photon polymerization exposure conditions at $405 \mathrm{~nm}$. A series of $10 \mu \mathrm{m}$ diameter circular phase elements is written using intensities ranging from $207 \mathrm{~mW} / \mathrm{cm}^{2}$ to $3442 \mathrm{~mW} / \mathrm{cm}^{2}$ and exposure times up to 2.5 seconds. This allows us to explore the response of the material over a wide range of exposure conditions. A summary of this data is shown in Figure 3 . The peak refractive index change for each phase element is plotted vs. intensity and exposure time for two material formulations: one containing $10 \mathrm{wt} \%$ of the synthesized monomer and one containing $30 \mathrm{wt} \%$ of the monomer. 

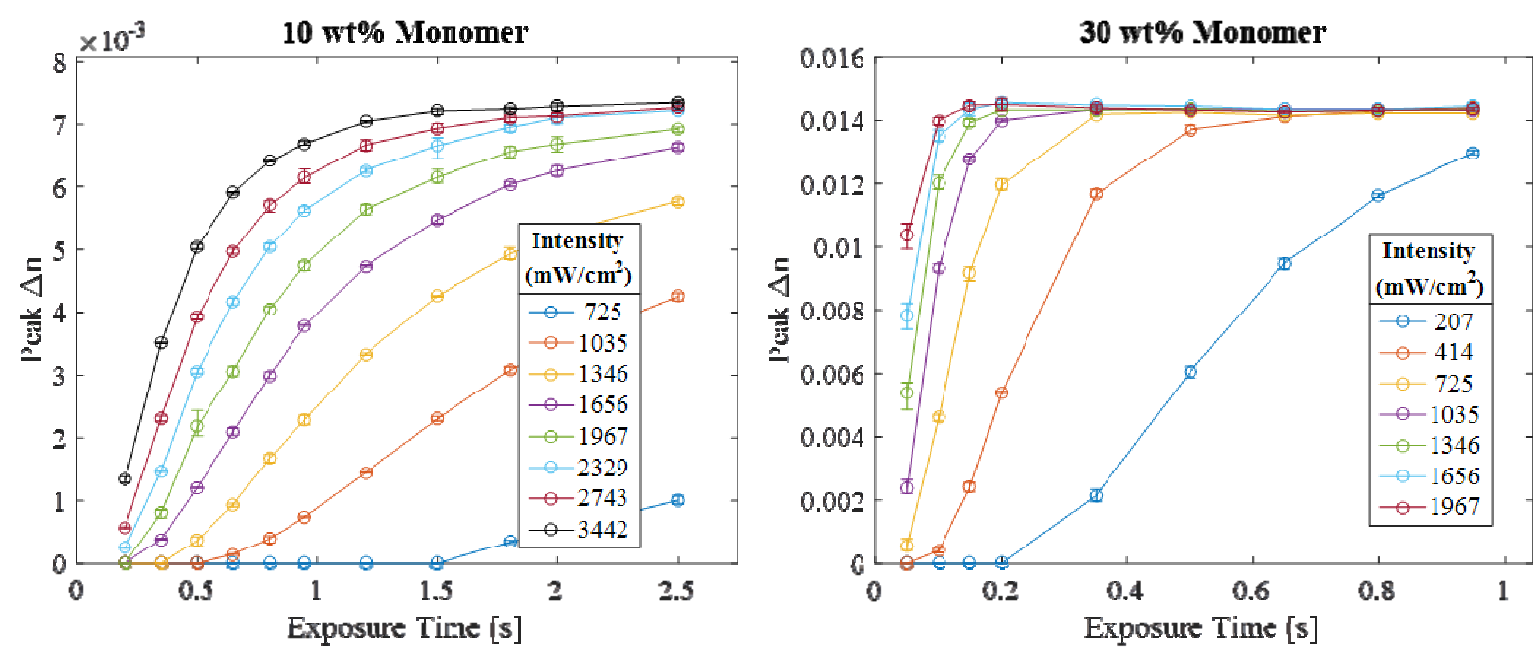

Figure 3. Peak measured index of refraction change vs. exposure time for a range of writing intensities. The graph on the left shows the material response for a photopolymer formulation with $10 \mathrm{wt} \%$ monomer, while the graph on the write shows the response for $30 \mathrm{wt} \%$ monomer. Both show a saturated index response, with the larger index response occurring in the material with the larger monomer concentration.

In both formulations, the index response saturates at high intensity and exposure times. The response in the $30 \mathrm{wt} \%$ monomer is much more sensitive, giving more index of refraction change for lower intensities and exposure times. The $30 \mathrm{wt} \%$ monomer formulation also results in a higher refractive index change, with a saturated value of 0.014 vs. the maximum value of 0.007 for the $10 \mathrm{wt} \%$ formulation. The index response can also be scaled by the intensity to show the linear response with dose $\left(\mathrm{mJ} / \mathrm{cm}^{2}\right)$ over the range of intensities explored here. This linear response with intensity is important for recording high fidelity holograms and other optical elements [19]. While the $30 \mathrm{wt} \%$ monomer index response is clearly independent of intensity used, the $10 \mathrm{wt} \%$ samples do demonstrate some dependence on the writing intensity. These samples also had lower concentrations of photoinitiator and we expect this response is due to the lower concentration of photoinitiator relative to the total concentration of oxygen in the sample.
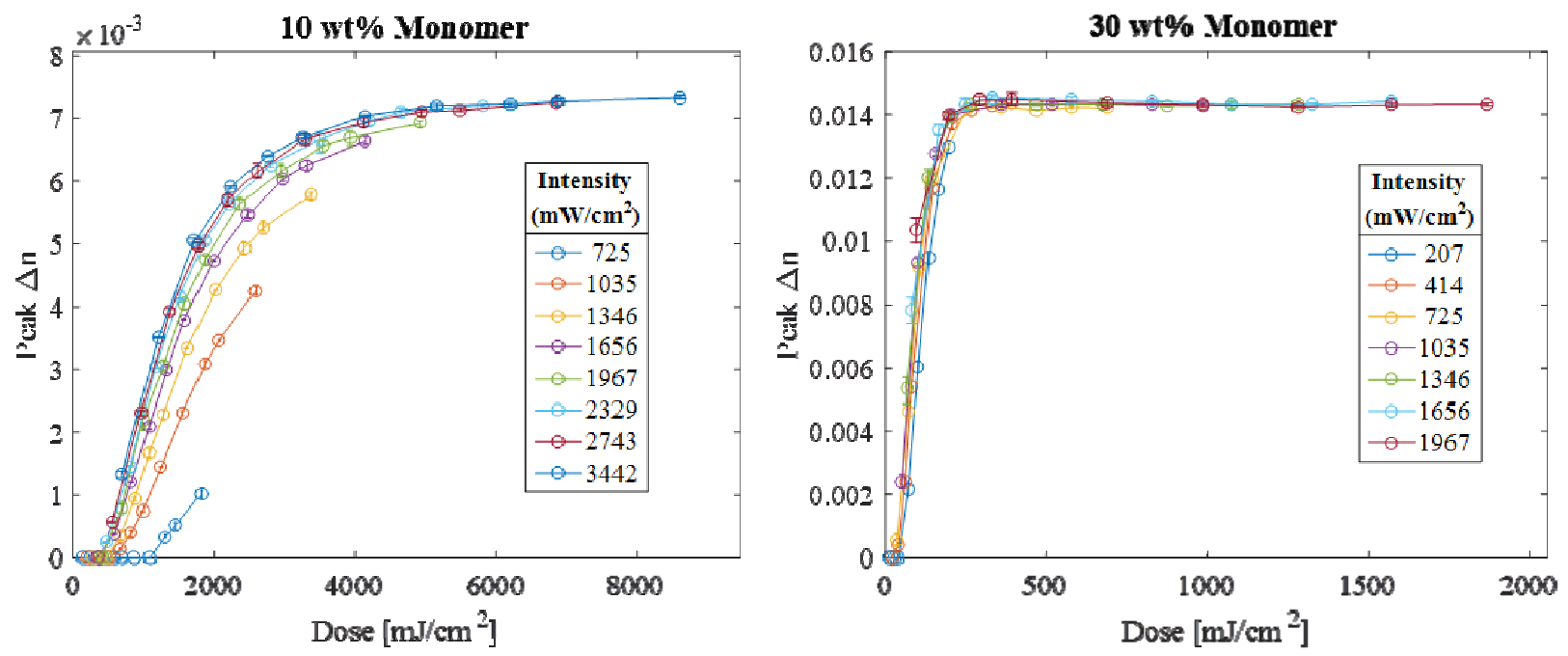

Figure 4: Peak measured refractive index change scaled by the exposure dose for a range of writing intensities.

\subsection{Two-photon Exposure}

The above experiment was repeated to explore the two-photon response in the $30 \mathrm{wt} \%$ writing monomer formulation and the results are shown in Figure 5. Fischer et al has shown that the polymerization threshold when using high repetition rate lasers $(>100 \mathrm{kHz})$ follows a mechanism that is consistent with two-photon absorption, whereas damage mechanisms 
due to thermal effects occur for repetition rates below $1 \mathrm{MHz}$ [20]. Because our $50 \mathrm{MHz}$ repetition rate laser operates well above the $1 \mathrm{MHz}$ regime, we should only be observing reactions produced by two-photon polymerization, and not by thermal accumulation. The scaling of the refractive index response curve by the effective dose of $I^{2} t$ in Figure 5 further demonstrates this conclusion. The deviation from this $I^{2} t$ dependence at lower intensities may again be due to the oxygen effects and needs to be investigated in more depth. While the intensities and response of the material are very different from the case of single photon polymerization, a careful study of the material allows us to achieve similar peak change of index of refraction, 0.012 , and map out the index response for a large range of exposure conditions. Twophoton polymerization can be used to create more localized structures in the photopolymer in $3 \mathrm{D}$, so understanding this response is important for designing complicated 3D structures.
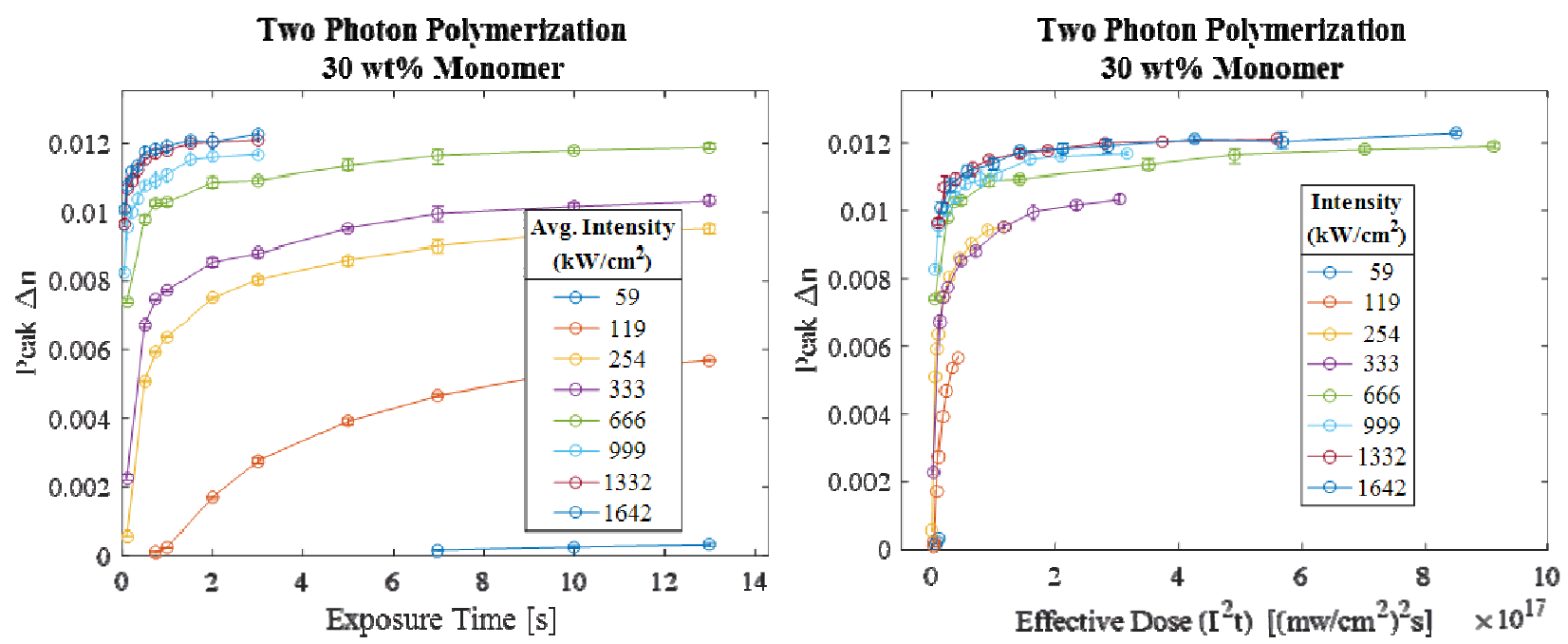

Figure 5. Peak measured index of refraction response vs. exposure time and effective dose using a $780 \mathrm{~nm}$ pulsed laser. This maps out the two-photon response of the photopolymer for the range of average intensities shown.

\subsection{Measuring Diffusion}

Because the optical path length difference in these photopolymer systems is formed through the diffusion of high refractive index monomer through the matrix, the characteristic diffusion time is an essential parameter in designing optical and material systems. This can be measured using the same writing and imaging methods described above. Once the saturation time for a given material is determined for a given intensity, a series of saturated spots is written into the material to deplete the local concentration of monomer. Over time, new monomer diffuses into these areas due to the monomer concentration gradient created by the first exposure. To measure the time over which this occurs, a second exposure is performed on top of the first after varying delay times and the final index of refraction is monitored for each delay time. This index of refraction can be related to the final concentration of monomer in the exposed spot. The concentration of monomer vs. delay time is fit to a single parameter diffusion model for diffusion into a cylinder [21] as shown in Figure 6 (left) giving a diffusion constant of $1.4 \times 10^{-2} \mu \mathrm{m}^{2} / \mathrm{s}$. This value allows us to calculate the time it takes for index structures of various sizes to develop in the material, an important design parameter.

The writing monomer used in these experiments is multi-functional, and so polymerization of this monomer is expected to result in a higher cross-linked material that will slow down diffusion of monomer into the polymerized areas. If a third exposure is performed, the diffusivity is shown to decrease by more than a factor of two (Figure 6 right). Understanding the change in diffusion time for multiple exposures is essential for multiplexed systems. 

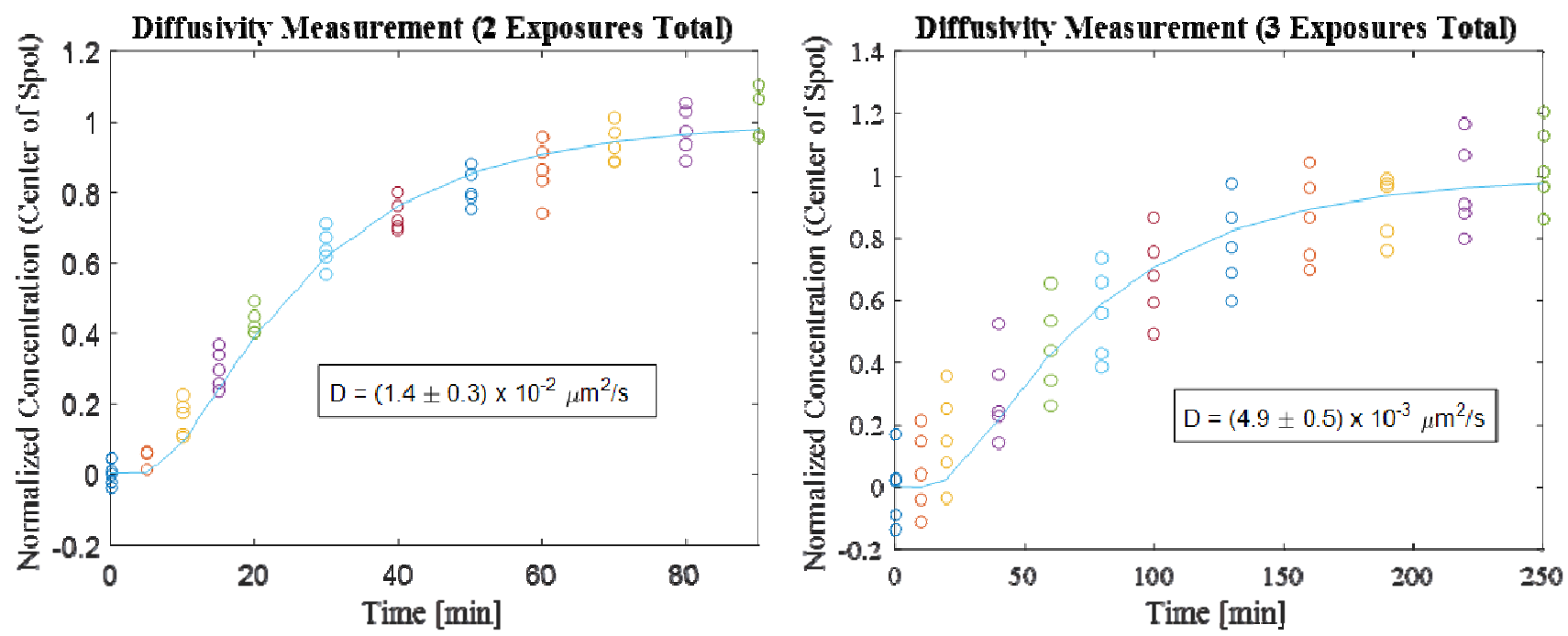

Figure 6. Normalized monomer concentration at the center of the exposed feature vs. delay time between writing steps. Data is shown along with the Fickian diffusion fit. On the left, the diffusivity fit parameter D is calculated for two exposures and on the right, diffusivity is calculated for three exposures.

\subsection{Multiple Write Exposure}

For a single exposure, the maximum achievable $\Delta \mathrm{n}$ in this material is 0.014 at $30 \mathrm{wt} \%$ monomer loading. However, as discussed in the diffusion measurements above, after an initial exposure depletes the local concentration of writing monomer, more monomer will diffuse into the exposed region. A second exposure can be used to polymerize this new monomer. Continued steps will increase the local concentration of writing monomer and polymer, increasing the local change in index of refraction. The measured $\Delta \mathrm{n}$ for multiple exposures of a saturation dose for the $30 \mathrm{wt} \%$ material is shown in Figure 7. Each exposure increases the local index of refraction until the response is fully saturated, likely due to decreased diffusion of new monomer into the highly-crosslinked material. Multiple exposures increase the achievable index of refraction contrast from 0.014 to 0.025 , a large enhancement of $\Delta \mathrm{n}$ from exposure conditions and no material changes.
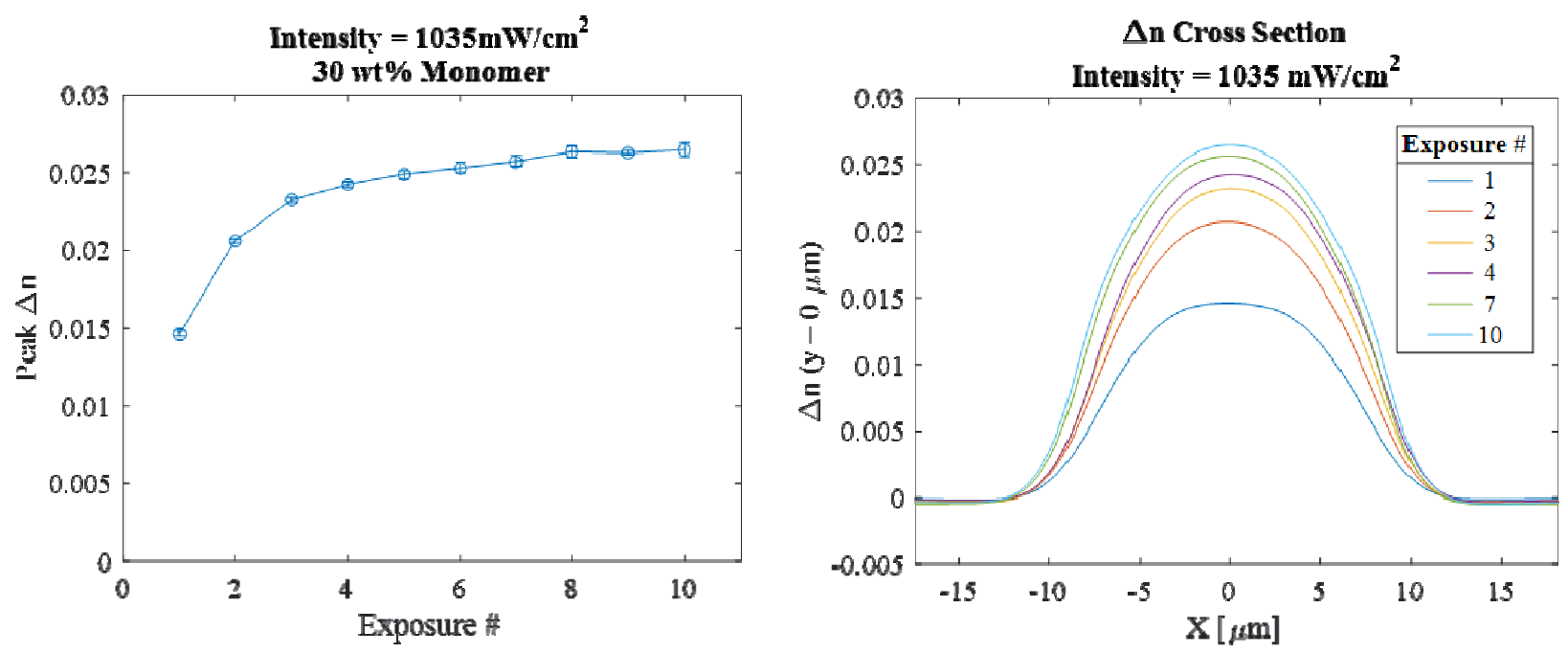

Figure 7. Increase in $\Delta \mathrm{n}$ achieved through multiple exposures. Each exposure is sufficient to locally saturate the index response. Diffusion of new monomer into the exposure region before subsequent exposures allows for a higher final index contrast. 


\section{CONCLUSION}

We apply the TIE phase imaging method to make quantitative, 2D measurements of the index profiles written into a twochemistry holographic photopolymer using a focused beam. The method is used to characterize both the single- and twophoton index response of the material over a wide range of exposure intensities as well as investigating the characteristic diffusion times as a function of polymerization. The method is straightforward to implement and is a powerful tool to investigate the index response and diffusion characteristics of different materials to optimize characteristics for the design of optical elements with low spatial frequency features. Utilizing these measurements and modifying the exposure conditions allows us to achieve an enhanced $\Delta n$ without changing any of the chemistry.

\section{REFERENCES}

[1] Li, Haoyu, Yue Qi, James P. Ryle, and John T. Sheridan. "Self-written waveguides in a dry acrylamide/polyvinyl alcohol photopolymer material." Applied optics 53, no. 34 (2014): 8086-8094.

[2] Urness, Adam C., Ken Anderson, Chungfang Ye, William L. Wilson, and Robert R. McLeod. "Arbitrary GRIN component fabrication in optically driven diffusive photopolymers." Optics express 23, no. 1 (2015): 264-273.

[3] Piao, Jing-Ai, Gang Li, Mei-Lan Piao, and Nam Kim. "Full color holographic optical element fabrication for waveguide-type head mounted display using photopolymer." Journal of the Optical Society of Korea 17, no. 3 (2013): 242-248.

[4] Akbari, Hoda, Izabela Naydenova, and Suzanne Martin. "Using acrylamide-based photopolymers for fabrication of holographic optical elements in solar energy applications." Applied optics 53, no. 7 (2014): 1343-1353.

[5] Berneth, Horst, Friedrich-Karl Bruder, Thomas Fäcke, Rainer Hagen, Dennis Hönel, David Jurbergs, Thomas Rölle, and Marc-Stephan Weiser. "Holographic recording aspects of high-resolution Bayfol® HX photopolymer." In Proc. SPIE, vol. 7957, p. 79570H. 2011.

[6] Teague, Michael Reed. "Deterministic phase retrieval: a Green's function solution." JOSA 73, no. 11 (1983): 14341441.

[7] Streibl, Norbert. "Phase imaging by the transport equation of intensity." Optics communications 49, no. 1 (1984): 610.

[8] Gureyev, T. E., A. Roberts, and K. A. Nugent. "Partially coherent fields, the transport-of-intensity equation, and phase uniqueness." JOSA A 12, no. 9 (1995): 1942-1946.

[9] Zuo, Chao, Qian Chen, Weijuan Qu, and Anand Asundi. "Noninterferometric single-shot quantitative phase microscopy." Optics letters 38, no. 18 (2013): 3538-3541.

[10] Roberts, Ann, Eric Ampem-Lassen, Anton Barty, Keith A. Nugent, Gregory W. Baxter, N. M. Dragomir, and S. T. Huntington. "Refractive-index profiling of optical fibers with axial symmetry by use of quantitative phase microscopy." Optics letters 27, no. 23 (2002): 2061-2063.

[11] Jesacher, A., P. S. Salter, and M. J. Booth. "Refractive index profiling of direct laser written waveguides: tomographic phase imaging." Optical Materials Express 3, no. 9 (2013): 1223-1232.

[12] Roelle, Thomas, Friedrich-Karl Bruder, Thomas Faecke, Marc-Stephan Weiser, Dennis Hoenel, and Nicolas Stoeckel. "Photopolymer compositions for optical elements and visual displays." U.S. Patent Application 12/569,184, filed September 29, 2009.

[13] Paganin, D., A. Barty, P. J. McMahon, and K. A. Nugent. "Quantitative phase-amplitude microscopy. III. The effects of noise." Journal of microscopy 214, no. 1 (2004): 51-61.

[14] Martinez-Carranza, J., K. Falaggis, and T. Kozacki. "Multi-filter transport of intensity equation solver with equalized noise sensitivity." Optics express 23, no. 18 (2015): 23092-23107.

[15] Waller, Laura, Lei Tian, and George Barbastathis. "Transport of intensity phase-amplitude imaging with higher order intensity derivatives." Optics express 18, no. 12 (2010): 12552-12561.

[16] Martinez-Carranza, J., K. Falaggis, and T. Kozacki. "Optimum plane selection for transport-of-intensity-equationbased solvers." Applied optics 53, no. 30 (2014): 7050-7058.

[17] Jingshan, Zhong, Rene A. Claus, Justin Dauwels, Lei Tian, and Laura Waller. "Transport of intensity phase imaging by intensity spectrum fitting of exponentially spaced defocus planes." Optics express 22, no. 9 (2014): 10661-10674. 
[18] Martinez-Carranza, Juan, Konstantinos Falaggis, and Tomasz Kozacki. "Solution to the Boundary problem for Fourier and Multigrid transport equation of intensity based solvers." Photonics Letters of Poland 7, no. 1 (2015): 24.

[19] Kowalski, Benjamin A., Adam C. Urness, Martha-Elizabeth Baylor, Michael C. Cole, William L. Wilson, and Robert R. McLeod. "Quantitative modeling of the reaction/diffusion kinetics of two-chemistry diffusive photopolymers." Optical Materials Express 4, no. 8 (2014): 1668-1682.

[20] Fischer, Joachim, Jonathan B. Mueller, Johannes Kaschke, Thomas JA Wolf, Andreas-Neil Unterreiner, and Martin Wegener. "Three-dimensional multi-photon direct laser writing with variable repetition rate." Optics express 21, no. 22 (2013): 26244-26260.

[21] Crank, J., [The Mathematics of Diffusion], Oxford University Press, London, 69-74 (1975). 